\title{
Zur Übersetzung des Manifests für eine Post-Kritische Pädagogik
}

Martin Bittner und Anke Wischmann

Die Übersetzung des englischsprachigen »Manifesto for a Post-Critical Pedagogy« (Hodgson et al. 2017) in die deutsche Sprache (Hodgson et al. 2022: 19) bildet den Ausgangspunkt dieses Bandes. Die versammelten Beiträge sind fast ausschließlich während einer Arbeitstagung im November 2020 am Zentrum für Bildungs-, Unterrichts-, Schul- und Sozialisationsforschung (ZeBUSS) ${ }^{1}$ der Europa-Universität Flensburg erstmals diskutiert und anschließend in die hier abgedruckten Texte überführt worden.

Das Manifest umreißt die Perspektive einer Post-Kritischen Pädagogik, indem fünf Grundsätze formuliert werden (vgl. Hodgson et al. 2022).

- Der erste Grundsatz besagt, dass es Grundsätze gibt, die es sich zu verteidigen lohnt.

- Der zweite Grundsatz reklamiert eine hermeneutische Perspektive - in Abgrenzung zu einer normativen.

- Der dritte Grundsatz unterstellt eine grundlegende Gleichheit.

- Der vierte Grundsatz konstatiert eine hoffnungsvolle Sichtweise (in Abgrenzung zu einer optimistischen).

1 Als Initiator des Vorhabens, möchte ich - Dr. phil. Martin Bittner - an dieser dieser Stelle dem Direktorium des ZeBUSS und Prof. Dr. Jürgen Budde für die ideelle und später auch finanzielle Untersützung danken. Prof. Dr. Anke Wischmann danke ich für die sehr gute gemeinsame Arbeit an diesem Projekt. 
- Der fünfte Grundsatz formuliert schließlich eine Verschiebung von der Bildung für Bürgerschaft zur Liebe für die Welt.

Diese Grundsätze vermitteln jene Perspektivierungen, die im Anspruch der Position der Post-Kritik stehen. Grundsätze »die gegründet sind auf der Überzeugung der Möglichkeit der Veränderung, wie sie in der kritischen Theorie und Pädagogik zu finden ist, allerdings mit einer affirmativen Haltung: eine post-kritische Ausrichtung von Bildung, die unter aktuellen Bedingungen an Einfluss gewinnen wird und dies wiederum ist begründet in einer Hoffnung für das, was noch kommen wird « (Hodgson et al. 2022: 20).

Grundsätze die in Form eines Manifests formuliert sind, unterstreichen die besondere Relevanz für eine gegenwärtige und zukünftige Perspektivierung sozialer und politischer Problemlagen und deren pädagogischer Bedeutsamkeit und Effekte. Die Problemlagen könnten für die Erziehungswissenschaft kaum vielfältiger sein, sie lassen sich in einem Spannungsfeld verorten: Einerseits einer globalen und international vergleichenden Bildungsforschung, die neoliberale Wirtschaftsweisen und Prinzipien als Notwendigkeit befördert und an eine evidenzbasierte Pädagogik koppelt ist; andererseits ist diesen Perspektivierungen eine Position vorangestellt, die die Eigenständigkeit, Freiheit, Vernunft und Aufklärung des Menschen durch Bildung unterstreicht, woraus eine Abgrenzung bspw. zu Kompetenzperspektiven innerhalb von Bildungsvorstellungen folgt - auch letztere Kritik und Entzauberung ist durch empirische Forschung vermittelt. Das Manifest mit seinen Grundsätzen wendet sich den letztgenannten Punkten flankierend zu. In seiner Utopie finden sich eine Reihe von Anklängen wissenschaftlicher Konzeptionen, die es zukünftig genauer auszudeuten gilt. Dazu bedarf es theoretischer und methodologischer Auseinandersetzungen ${ }^{2}$

2 Dass solche Auseinandersetzungen selbst wieder in Manifesten gründen können bzw. aus anderen Manifesten hervorgehen, sei hier mit Verweis auf zwei ältere Manifeste erwähnt: siehe Willis \& Trondman (2000) sowie Biesta \& Säfström (2011). 
mit dem Begriff der Post-Kritik für die Erziehungswissenschaft umzugehen sowie der Reflexion der spezifischen Wissensgenerierung, wie sie durch die Übersetzung von Texten vor dem Hintergrund eines globalen Anspruchs entsteht. Das Manifest für eine Post-Kritische Pädagogik ist bereits in englischer (Hodgson et al. 2017), spanischer (Hodgson et al. 2020) türkischer (Hodgson et al. 2021) und mit diesem Band in deutscher (Hodgson et al. 2022) Übersetzung verfügbar.

Entsprechend soll an dieser Stelle einführend nach dem »wozu« der Übersetzung gefragt werden. $\mathrm{Zu}$ allgemeinen Fragen der Übersetzung wird am prominentesten Walter Benjamin rezipiert, der im Jahr 1921 mit Blick auf seine Übersetzung Baudelaires die Aufgabe des Übersetzers formuliert hatte (Benjamin 1972). Dabei fragt er, ob »[.] eine Übersetzung den Lesern, die das Original nicht verstehen« gälte (Benjamin 1972: 9) oder ob die Übersetzung der »einzig mögliche Grund [sei], >Dasselbe< wiederholt zu sagen « (Benjamin 1972: 9)?

Mit diesen Fragen muss sich auch die vorliegende Übersetzung des Manifests konfrontiert sehen. Sie entsprang zunächst aus einem hochschuldidaktischen Ansinnen von Martin Bittner, Lehramtsstudierenden eines Pädagogikseminars die Idee der Post-Kritischen Pädagogik nahe $\mathrm{zu}$ bringen - womit die erste Frage nach dem Verstehen des Originals, wenn auch in einer Ambivalenz, beantwortet wäre. Von Studierenden wird qua Zulassung mit dem Abitur ein umfassendes Sprachniveau des Englischen vorausgesetzt und damit die Fähigkeit verbunden, englischsprachige wissenschaftliche Texte im Rahmen des Studiums rezipieren zu können. Dennoch zeichnen sich in universitären Seminaren immer wieder Schwierigkeiten im Verstehen englischsprachiger Texte ab, Studierende bemühen dabei selbst den Ansatz der Übersetzung, um das Original zu verstehen. Die Vermittlung der theoretischen Positionen des Manifests explizit durch eine Übersetzung vorzunehmen, wurde daher als ein Modus der Aneignung und Auseinandersetzung mit dem Text konzipiert und galt dem Versuch, die Post-Kritische Pädagogik in seiner Relevanz zu diskutieren.

Mit Blick auf die zweite Frage Benjamins - der Suche nach einem Grund, dasselbe nochmal sagen zu können - bedarf es einer Positionierung: In dem vorliegenden Sammelband geht es nicht darum, dasselbe 
wiederholt zu sagen. Bereits in unseren Anfragen zu der Arbeitstagung wurden wir von einigen Kolleg*innen an dieser Stelle missverstanden. Es geht nicht darum, das Manifest "feiern« zu wollen, oder »dasselbe« noch einmal (auf Deutsch) zu sagen. Originaltexte und Übersetzungen erscheinen immer auch in einem sozialen und kulturellen Kontext (einem Wechselspiel aus Diskursen und Praktiken), dies gilt es nicht nur zu berücksichtigen, sondern post-kritisch zu erkennen und anzuerkennen. Somit wurden die Autor*innen für die Auseinandersetzung mit dem Manifest nicht darum gebeten, »dasselbe« mit Bezug auf die deutschsprachige Fassung des Manifests herauszustellen. Eine solche Bezugnahme ginge einher mit einer Frage nach adäquaten Ausführungen oder einer »richtigen « Auslegung, die immer auch eine Abgrenzung erzeugen, wie auch die Frage nach dem Äquivalenten als eine Annäherung an das oder gar eine Einverleibung des Manifests schlichtweg nicht Ziel der Übersetzung sein kann. Es geht nicht darum, dass die Autor*innen und Herausgeber*innen am Ende diesem Manifest zustimmen wollen. Vielmehr wollen wir eine Debatte führen.

Tyson E. Lewis (2017) hat bereits in der Diskussion des Originals darauf hingewiesen, dass es sich bei dem Text von Hodgson et al. (2017) vielleicht eher um eine Erklärung, als um ein Manifest handelt. Dabei stellt er heraus, dass es fraglich wäre, ob die Geste des Unterschreibens überhaupt eine angemessene Antwort darstellen würde. Ob nun Manifest oder Erklärung, die Debatte über Fragen von Kritik und PostKritik in der Pädagogik und Erziehungswissenschaft wird weiter vorangebracht, wodurch die Übersetzung eine Würdigung der Debatte darstellt, auch insofern, dass sich in der Übersetzung auch die Qualität eines Textes zeigt, indem er überhaupt übersetzbar ist. Bei einer einfachen Zustimmung stehen zu bleiben hieße, das Anregungspotential des Manifests zu ignorieren.

»Übersetzung ist eine Form. Sie als solche zu fassen, gilt es zurückzugehen auf das Original. Denn in ihm liegt deren Cesetz als in dessen Übersetzbarkeit beschlossen.«(Benjamin 1972: 9)

Die Übersetzung gewinnt ihre Eigenständigkeit, ohne dabei das Original in gleicher Weise zu erfassen, es ist ein Überleben, ein Fortleben 
- jedoch nicht nur in einem passiven Sinne, sondern insofern, dass dadurch auch das Original eine umfassende Entfaltung erfährt. Eben diese Eigenständigkeit, dieses Öffnen, Ausdehnen und Ausbrechen des Manifests gilt es zu diskutieren und in diesem Buch zu erkunden, im Sinne einer deutschsprachigen Verortung des Manifests auch als eine Ergänzung bzw. Ermöglichung von Erkenntnis - die ja immer nur auch vorläufige Erkenntnis sein kann.

\section{Literatur}

Benjamin, Walter (1972): »Die Aufgabe des Übersetzers«, in: Walter Benjamin (Hg.): Gesammelte Schriften. Unter Mitarbeit von Rolf Tiedemann, Tillman Rexroth, Hermann Schweppenhäuser, Theodor W. Adorno und Gershom Scholem. Frankfurt a.M.: Suhrkamp (Suhrkamp Taschenbuch Wissenschaft, 934,1): S. 9-21.

Biesta, Gert; Säfström, Carl Anders (2011): »A Manifesto for Education", in: Policy Futures in Education 9 (5): S. 540-547. DOI: 10.2304/pfie.2011.9.5.540.

Hodgson, Naomi; Vlieghe, Joris; Zamojski, Piotr (2017): »Manfiesto for a Post-Critical Pedagogy«, in: Naomi Hodgson, Joris Vlieghe und Piotr Zamojski (Hg.): Manifesto for a post-critical pedagogy. Brooklyn NY: punctum books: S. 15-19.

Hodgson, Naomi; Vlieghe, Joris; Zamojski, Piotr (2020): »Manifiesto por una pedagogía post-crítica (traducción al español)«, in: Teor. educ. 32 (2): S. 7-11. DOI: 10.14201/teri.22862.

Hodgson, Naomi; Vlieghe, Joris; Zamojski, Piotr (2021): »Eleştirel Pedagoji Sonrası İçin Manifesto«, in: eleştirel pedagoji - Journal of Critical Pedagogy (67): S. 99-101.

Hodgson, Naomi; Vlieghe, Joris; Zamojski, Piotr (2022): »Manifest für eine Post-Kritische Pädagogik«, in: Martin Bittner, Anke Wischmann (Hg.): Kritik und Post-Kritik. Zur deutschsprachigen Rezeption des »Manifests für eine Post-Kritische Pädagogik«. Bielefeld: transcipt Verlag: S. 19-24. 
Lewis, Tyson E. (2017): »A Response to the »Manifesto for a Post-Critical Pedagogy«, in: Naomi Hodgson, Joris Vlieghe und Piotr Zamojski (Hg.): Manifesto for a post-critical pedagogy. Brooklyn NY: punctum books: S. 23-34.

Willis, Paul; Trondman, Mats (2000): »Manifesto for Ethnography«, in: Ethnography 1 (1): S. 5-16. DOI: 10.1177/14661380022230679. 